optimally treat RLS in dialysis patients. We conducted a systematic review to identify therapies to reduce the severity of RLS.

Methods We systematically searched MEDLINE and EMBASE and hand searched narrative reviews for randomized controlled trials that assessed the use of pharmacological or nonpharmacological interventions to reduce the severity of RLS among patients receiving dialysis. The primary outcome was RLS symptoms.

Results Of 621 abstracts identified, 12 studies that included 13 interventions met the inclusion and exclusion criteria. All trials reported interventions that significantly improved RLS severity. The interventions included dopamine agonists $(n=6)$, gabapentin $(n=2)$, intradialytic exercise $(n=2)$ intravenous iron $(n=1)$, vitamins $C$ and $E(n=1)$, and clonidine $(n=1)$. In studies that compared two interventions, ropinirole gabapentin, and intradialytic exercise with resistance were all more effective than L-dopa in comparisons. However, all studies were at high risk of bias with the most common causes due to lack of allocation concealment, high losses to follow-up, and missing data. Further, follow-up for these studies was short-term ranging from 3 days to 24 weeks.

Conclusions Ropinirole, gabapentin, and intradialytic exercise appear promising to reduce RLS in dialysis patients. However, methodologically rigorous, appropriately powered, longer term RCTs are required to determine the optimal treatment strategy for RLS among dialysis patients.

\section{THE EFFECTIVENESS OF PHARMACOLOGICAL AND NON-PHARMACOLOGICAL INTERVENTIONS IN IMPROVING RESTLESS LEGS SYNDROME SEVERITY AMONG PATIENTS ON DIALYSIS: A SYSTEMATIC REVIEW}

K Quach, M Gandhi, KS Brimble, M Walsh. McMaster University, Hamilton, Ontario

10.1136/jech-2014-205217.2

Background and objectives Restless legs syndrome (RLS) is common amongst patients receiving dialysis. It is unclear how to 